\title{
Representação política atual do idoso e o reflexo sobre uma instituição de ensino superior pública
}

Currente political representation of the elderly and the reflection on a public higher institution

Representación política actual de lós ancianos y la reflexión sobre uma institución pública de educatión superior Guilherme Higuchi KATO ${ }^{1}$

Maria Cristina Rosifini ALVES REZENDE ${ }^{2}$

Laís Maria PESCININI-E-SALZEDAS ${ }^{3}$

Maria Isabel Rosifini ALVES REZENDE ${ }^{4}$

Letícia Maria PESCININI-SALZEDAS ${ }^{3}$

Leda Maria Pescinini SALZEDAS ${ }^{\mathbf{5}}$

${ }^{1}$ Acadêmico de Odontologia. UNESP Univ Estadual Paulista, Faculdade de Odontologia de Araçatuba, Departamento de Diagnóstico e Cirurgia 16015-050 Araçatuba-SP, Brasil

${ }^{2}$ Docente. UNESP Univ Estadual Paulista, Faculdade de Odontologia de Araçatuba, Departamento de Materiais Odontológicos e Prótese 16015-050 Araçatuba-SP, Brasil ${ }^{3}$ Acadêmica de Medicina, Faculdade de Medicina de Marília da Universidade de Marília (UNIMAR) 17525-902 Marília-SP, Brasil

${ }^{4}$ Mestranda, Programa de Pós-Graduação Stricto Sensu da Faculdade de Direito de Ribeirão Preto da Universidade De São Paulo (USP) 14040-906 Ribeirão Preto-SP, Brasil ${ }^{5}$ Docente. UNESP Univ Estadual Paulista, Faculdade de Odontologia de Araçatuba, Departamento de Diagnóstico e Cirurgia 16015-050 Araçatuba-SP, Brasil

\section{Resumo}

Introdução: $\mathrm{O}$ aumento da expectativa de vida da população tem contribuído para a mudança na estrutura demográfica brasileira e aos indivíduos mais velhos da sociedade estão sendo atribuídos novos papéis sociais. Para efeito legal, idoso é a denominação oficial de todos os indivíduos que tenham sessenta anos de idade ou mais. Os "velhos jovens" são as pessoas com idade entre sessenta e oitenta anos, caracterizadas por um envelhecimento ativo e independente, voltado para a integração e a autogestão. Avalia-se que aumento da expectativa de vida leva a um interesse maior dos idosos pela política, e que elas representam uma geração de pessoas que viveram a política mais intensamente. Objetivo: O objetivo deste trabalho foi avaliar a participação de políticos com mais de 60 anos de idade nas representações municipal, estadual e federal, e seus reflexos sobre a Faculdade de Odontológica de Araçatuba-UNESP. Material e método: Foi desenvolvida busca nos relatórios das últimas eleições (2016 e 2018) na cidade de Araçatuba e nos estados da Região Sudeste. Resultados: Verificou-se que líderes municipais e estaduais de São Paulo e o presidente são idosos. No Estado de São Paulo 30,2\% são políticos idosos, e Região Sudeste são 24,16\%. Em ambos, há maior representação na esfera municipal (39,19\% e 28,88\%). A faixa etária de 60 anos ou mais, na esfera municipal no Estado de São Paulo e no Sudeste, está entre as mais frequentes com valor similar a faixa de 50 a 59 anos; e nas esferas estadual e federal ocupa a terceira posição. Conclusão: Conclui-se que o idoso tem desenvolvido um papel atuante na política, ocupando cerca de um terço das representações, em especial na esfera municipal.

Descritores: Idoso; Política; Capacidade de Liderança e Governança; Brasil

\section{Abstract}

Introduction: The increase in life expectancy of the population has contributed to the change in the demographic structure in Brazil and older individuals in society are being assigned new social roles. For legal purposes, elderly is the official name of all individuals who are sixty years of age or older. The "old young people" are people aged between sixty and eighty, characterized by active and independent aging, focused on integration and self-management. It is estimated that an increase in life expectancy leads to a greater interest of the elderly in politics, and that they represent a generation of people who have lived politics more intensely. Objective: The objective of this work was to evaluate the participation of politicians over 60 years of age in the municipal, state and federal representations, and their reflections on the Faculty of Dentistry of Araçatuba-UNESP. Material and method: A search was developed in the reports of the last elections (2016 and 2018) in the city of Araçatuba and in the states of the Southeast Region. It was found that municipal and state leaders in São Paulo and the president are elderly. Results: In the State of São Paulo, 30.2\% are elderly politicians, and the Southeast Region is $24.16 \%$. In both, they have greater representation in the municipal sphere (39.19\% and 28.88\%). The age group of 60 years or more, in the municipal sphere in the State of São Paulo and in the Southeast, is among the most frequent ones with a value similar to the range of 50 to 59 years; and at the state and federal levels it occupies the third position. Conclusion: It is concluded that the elderly have developed an active role in politics, occupying about one third of the representations, especially in the municipal sphere.

Descriptors: Aged; Politics; Leadership and Governance Capacity; Brazil

\section{Resumen}

Introducción: El aumento de la esperanza de vida de la población ha contribuido al cambio en la estructura demográfica en Brasil y a las personas mayores de la sociedad se les está asignando nuevos roles sociales. Para fines legales, ancianos es el nombre oficial de todas las personas que tienen sesenta años de edad o más. Los "jóvenes de edad" son personas de entre sesenta y ochenta años, caracterizados por un envejecimiento activo e independiente, centrados en la integración y la autogestión. Se estima que un aumento en la esperanza de vida conduce a un mayor interés de los ancianos en la política, y que representan a una generación de personas que han vivido la política más intensamente. Objetivo: El objetivo de este trabajo fue evaluar la participación de políticos mayores de 60 años en las representaciones municipales, estatales y federales, y sus reflexiones sobre la Facultad de Odontología de Araçatuba-UNESP. Material y método: Se desarrolló una búsqueda en los informes de las últimas elecciones (2016 y 2018) en la ciudad de Araçatuba y en los estados de la Región Sudeste, y se descubrió que los líderes municipales y estatales en São Paulo y el presidente son ancianos. Resultados: En el Estado de São Paulo, el 30,2\% son políticos de edad avanzada, y la región sudeste es el 24,16\%. En ambos, tienen mayor representación en el ámbito municipal $(39,19 \%$ y 28,88\%). El grupo de edad de 60 años o más, en el ámbito municipal en el Estado de São Paulo y en el sudeste, se encuentra entre los más frecuentes con un valor similar al rango de 50 a 59 años; y a nivel estatal y federal ocupa la tercera posición. Conclusión: Se concluye que los ancianos han desarrollado un papel activo en la política, ocupando aproximadamente un tercio de las representaciones, especialmente en el ámbito municipal.

Descriptores: Anciano; Política; Capacidad de Liderazgo y Gobernanza; Brasil

\section{INTRODUÇÃO}

Idoso é a denominação oficial de todos os indivíduos que tenham sessenta anos de idade ou mais, que é utilizada pela Organização Mundial de Saúde (OMS) e pelas políticas sociais que focalizam o envelhecimento, como exemplo, a Política Nacional do Idoso (PNI). Esse é o critério adotado para fins de censo demográfico ${ }^{1}$.
A expressão Terceira Idade, surgiu na França, a partir de 1962, em virtude da introdução de uma política de integração social da velhice visando à transformação da imagem das pessoas envelhecidas ${ }^{2}$. Terceira Idade é, então, a nova fase da vida entre a aposentadoria e o envelhecimento, caracterizada por um envelhecimento ativo e independente, voltado 
para a integração e a autogestão. Constitui um segmento geracional dentro do universo de pessoas consideradas idosas, ou seja, são os "velhos jovens" com idade entre sessenta e oitenta anos ${ }^{1}$. Segundo projeções do $\mathrm{IBGE}^{3}$, em 2030 a população brasileira terá 13,54\% dos indivíduos com 65 anos ou mais de idade, e no Estado de São Paulo serão 15,07\%. Dados do Tribunal Superior Eleitoral ${ }^{4}$ mostram que $8,2 \%$ do eleitorado brasileiro apto a votar nas eleições de 2018 estavam acima dos 70 anos. Dos 16,7 milhões de eleitores que são filiados a partidos políticos, $13 \%$ (2,2 milhões) estavam acima dos 70 anos. Avalia-se que o aumento da expectativa de vida leva a um interesse maior dos idosos pela política, e que elas representam uma geração de pessoas que viveram a política mais intensamente. Em 2015, 62\% dos parlamentares estavam acima dos 50 anos (Câmara-59\% e Senado-87\%) ${ }^{5}$. A faixa etária dos candidatos nas eleições de 2018 mostrou maior concentração na faixa etária dos 45 a 49 anos $(16,1 \%)$, seguida pelos candidatos entre 50 e 54 anos $(15,88 \%)$. O objetivo deste trabalho foi avaliar a participação de políticos com mais de 60 anos de idade nas representações municipal, estadual e federal, e seus reflexos sobre a Faculdade de Odontologia de Araçatuba-UNESP.

\section{MATERIAL E MÉTODO}

Trata-se de uma pesquisa descritiva e documental, desenvolvida com o intuito de avaliar a participação de políticos com mais de 60 anos de idade nas representações municipal, estadual e federal, com reflexos na Faculdade de Odontologia de Araçatuba-UNESP. Foi desenvolvida busca nos relatórios do Tribunal Superior Eleitoral ${ }^{6}$ e sites da Agência Câmara de Notícias $^{7}$, Agência Senado ${ }^{8}$, Gazeta do Povo ${ }^{9,10}$, Senado Federal ${ }^{11}$ das últimas eleições (presidente e deputados: 2018; municipais: 2016) referente às representações na cidade de Araçatuba e nos estados que compõem a região sudeste (São Paulo, Rio de Janeiro, Espírito Santo e Minas Gerais), incluindo: Prefeito/Vice-Prefeito, Vereadores, Governador/Vice, Deputados Estaduais, Deputados Federais, Senadores, Presidente/VicePresidente.

Para facilitar a análise dos dados, os mesmos foram tabulados, estado a estado, nas esferas políticas analisadas, de acordo com o grupo etário do candidato eleito.

Os dados coletados foram tabulados, realizada estatística descritiva através da obtenção das distribuições de frequências absolutas e percentuais, e apresentadas sob a forma de tabelas.

\section{RESULTADOS}

Ao analisar os dados, considerou-se como representação municipal: Prefeito/Vice e Vereadores; representação estadual: Governador/Vice e Deputado Estadual; representação federal: Deputado Federal,
Senador e Presidente/Vice. Na análise do Estado de São Paulo, verificou-se que a participação de políticos com mais de 60 anos correspondeu 30,2\% dos 245 parlamentares do Estado de São Paulo, sendo que a maior participação dos idosos ocorreu na esfera municipal com 39,19\%, e representações similares nas esferas estadual e federal $(26,04 \%$ e $26,67 \%$, respectivamente) (Tabelas 1 e 2 ).

A faixa etária dos parlamentares eleitos em 2016 e 2018, no Estado de São Paulo, mostrou maior concentração na faixa etária dos 50 a 59 anos $(31,43 \%)$, seguida pelos parlamentares entre 60 a 69 anos $(22,04 \%)$ e entre 40 e 49 anos $(19,18 \%)$ (Tabela 1). Sendo que se considerarmos a faixa etária de 60 anos ou mais, correspondem a 30,20\%, com valor próximo ao primeiro em frequência.

Tabela 1- Distribuição da representação no Estado de São Paulo nas esferas municipal, estadual e federal, segundo faixa etária do parlamentar. ESTADO SÃO PAULO/IDADE $\quad 20-29 \quad 30-39 \quad 40-49 \quad 50-59 \quad 60-69 \quad 70-80+\quad$ TOTAL \begin{tabular}{lll} 
Prefeito/Vice & & \\
\hline Aracatuba & 2 & 2
\end{tabular} Araçatuba São Paulo-SP

Câmara municipal

Araçatuba

São Paulo-SP Governador/Vice São Paulo São Paulo

Deputados Federais

São Paulo

Senadores

São Paulo

Presidência/Vice

TOTAL
TORCENAG PORCENTAGEM

Fonte: Elaborada pelos autores (2020)

Tabela 2- Distribuição, numérica e porcentual, da participação dos parlamentares idosos em relação ao total de representantes nas esferas municipal, estadual e federal, no Estado de São Paulo.

\begin{tabular}{lccc}
\hline ESTADO SÃO PAULO & Total representantes & $60+$ & $\%$ \\
\hline MUNICIPAL & 74 & 29 & 39,19 \\
\hline ESTADUAL & 96 & 25 & 26,04 \\
\hline FEDERAL & 75 & 20 & 26,67 \\
\hline TOTAL & 245 & 74 & 30,2 \\
\hline
\end{tabular}

Fonte: Elaborada pelos autores (2020)

$\mathrm{Na}$ análise da Região Sudeste, verificou-se que a participação de políticos com mais de 60 anos correspondeu $24,16 \%$ dos 658 parlamentares de toda a região, sendo que a maior participação dos idosos ocorreu na esfera municipal com 28,88\%, e representações similares nas esferas estadual e federal $(21,86 \%$ e $23,32 \%$, respectivamente) (Tabelas 3 a 6).

$\mathrm{Na}$ Região Sudeste, a faixa etária dos parlamentares eleitos em 2016 e 2018, mostrou: na esfera municipal maior concentração na faixa etária dos 50 a 59 anos $(28,88 \%)$, seguida pelos parlamentares entre 40 e 49 anos $(25,67 \%)$ e entre 60 e 69 anos $(20,85 \%)$ (Tabela 3); na esfera estadual maior concentração na faixa etária dos 50 a 59 $\operatorname{anos}(28,67 \%)$, seguida pelos parlamentares entre 40 e $49 \operatorname{anos}(25,44 \%)$ e entre 30 e 39 anos $(20,79 \%)$ (Tabela 4); na esfera federal maior concentração na faixa etária dos 50 a 59 anos $(30,21 \%)$, seguida pelos parlamentares entre 40 e 49 anos $(26,94 \%)$ e entre 60 e 69 anos $(17,62 \%)$ (Tabela 5). 
Considerando como faixa etária 60 anos ou mais, na esfera municipal este grupo tem participação de $28,88 \%$, igual ao mais frequente (50 a 59 anos); nas esferas estadual e federal ocupa a terceira posição em frequência, com $21,86 \%$ e $23,32 \%$ respectivamente (Tabelas 3 a 5). Se considerarmos todas as esferas juntas, a faixa etária 60 anos ou mais constitui $24,28 \%$ dos representantes, e a faixa etária de 50 a 59 anos corresponde a $29,18 \%$.

Tabela 3- Distribuição da representação na Região Sudeste na esfera municipal, segundo faixa etária do parlamentar.

\begin{tabular}{llllllll}
\hline SUDESTE/MUNICIPAL & $20-29$ & $30-39$ & $40-49$ & $50-59$ & $60-69$ & $70-80+$ & TOTAL
\end{tabular} \begin{tabular}{lrr}
\hline Prefeito-Vice & & \\
\hline Araçatuba & 2 & 2 \\
\hline
\end{tabular}

São Paulo-SP

Rio de Janeiro-RJ

Vitória-ES

\begin{tabular}{llll}
\hline Câmara municipal & 1 & 1 & 2 \\
\hline
\end{tabular}

\begin{tabular}{llllll}
\hline Araçatuba & 1 & 4 & 6 & 4 & 15 \\
\hline
\end{tabular}

\begin{tabular}{llllllll}
\hline Arąacatuba & & 1 & 4 & 6 & 4 & & 15 \\
\hline & 2 & 7 & 7 & 17 & 16 & 6 & 55 \\
\hline
\end{tabular}

\begin{tabular}{lllllllll}
\hline Rio de Janeiro-RJ & 1 & 7 & 18 & 12 & 6 & 7 & 51 \\
\hline Belo Horizonte-MG & 1 & 6 & 12 & 14 & 7 & 1 & 41
\end{tabular}

\begin{tabular}{lcccccccc}
\hline Belo Horizonte-MG & 1 & 6 & 12 & 14 & 7 & 1 & 41 \\
\hline Vitória-ES & & 5 & 5 & 4 & 1 & & 15 \\
\hline \multicolumn{1}{c}{ TOTAI } & 4 & 27 & 48 & 54 & 39 & 15 & 187 \\
\hline
\end{tabular}

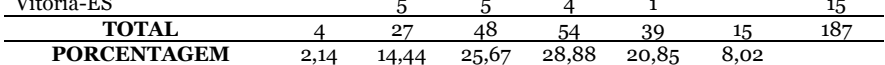

Fonte: Elaborada pelos autores (2020)

Tabela 4- Distribuição da representação na Região Sudeste na esfera

estadual, segundo faixa etária do parlamentar.

\begin{tabular}{llllllll}
\hline SUDESTE/ESTADUAL & $20-29$ & $30-39$ & $40-49$ & $50-59$ & $60-69$ & $70-80+$ & TOTAL
\end{tabular}

Governador

São Paulo

São Paulo

\begin{tabular}{l} 
Rio de Janeiro \\
\hline Minas Gerais
\end{tabular}

\begin{tabular}{l} 
Minas Gerais \\
\hline Espírito Santo
\end{tabular}

Deputados estaduais

São Paulo

Rio de Janeiro

Minas Gerais

$\begin{array}{r}\text { Espírito Santo } \\ \text { TOTAL } \\ \hline\end{array}$

PORCENTAGEM

Fonte: Elaborada pelos autores (2020)

Tabela 5- Distribuição da representação na Região Sudeste na esfera federal, segundo faixa etária do parlamentar.

\begin{tabular}{|c|c|c|c|c|c|c|c|}
\hline SUDESTE/FEDERAL & $20-29$ & $30-39$ & $40-49$ & $50-59$ & $60-69$ & $70-80+$ & TOTAL \\
\hline \multicolumn{8}{|l|}{ Deputados Federais } \\
\hline São Paulo & 4 & 10 & 15 & 24 & 14 & 3 & 70 \\
\hline Rio de Janeiro & 1 & 8 & 16 & 13 & 5 & 3 & 46 \\
\hline Minas Gerais & 1 & 12 & 17 & 11 & 10 & 2 & 53 \\
\hline Espírito Santo & 1 & & 2 & 4 & 3 & & 10 \\
\hline \multicolumn{8}{|l|}{ Senadores } \\
\hline São Paulo & & & & 2 & & 1 & 3 \\
\hline Rio de Janeiro & & 1 & & 1 & & 1 & 3 \\
\hline Minas Gerais & & & 1 & 2 & & & 3 \\
\hline Espírito Santo & & & 1 & 1 & & 1 & 3 \\
\hline \multicolumn{8}{|l|}{$\mathrm{m}$} \\
\hline Presidência/Vice & & & & & 2 & & 2 \\
\hline TOTAL & 7 & 31 & 52 & 58 & 34 & 11 & 193 \\
\hline PORCENTAGEM & 3,63 & 16,06 & 26,94 & 30,05 & 17,62 & 5,70 & \\
\hline
\end{tabular}

Fonte: Elaborada pelos autores (2020)

Tabela 6- Distribuição, numérica e porcentual, da participação dos parlamentares idosos em relação ao total de representantes nas esferas municipal, estadual e federal, na Região Sudeste.

\begin{tabular}{lccc}
\hline SUDESTE & Total de representantes & $\mathbf{6 0}+$ & $\mathbf{\%}$ \\
\hline MUNICIPAL & 187 & 54 & 28,88 \\
\hline ESTADUAL & 279 & 61 & 21,86 \\
\hline FEDERAL & 193 & 45 & 23,32 \\
\hline TOTAL & 659 & 160 & 24,28 \\
\hline
\end{tabular}

Fonte: Elaborada pelos autores (2020)

Dos parlamentares eleitos no estado de São Paulo em 2016(municipal) e 2018 (estadual e federal): $61,63 \%$ estão acima de 50 anos, sendo na esfera municipal verificada a maior porcentual de parlamentares acima de 50 anos com 70,27\% da representação, seguida pela federal $(61,34 \%)$ e estadual (55,2\%) (Tabela 7).

Dos parlamentares eleitos na região Sudeste em 2016(municipal) e 2018 (estadual e federal):
$53,41 \%$ estão acima de 50 anos, sendo na esfera municipal verificada a maior porcentual de parlamentares acima de 50 anos com 57,75\% da representação, seguida pela federal $(53,37 \%)$ e estadual $(50,54 \%)$ (Tabela 8$)$.

Dos parlamentares eleitos da Região Sudeste em 2018, 53,41\% dos parlamentares estavam acima dos 50 anos (Câmara Estadual-50,18\%, Câmara Federal -51,67\% e Senado-75\%).(Tabela 9).

Tabela 7- Distribuição, numérica e porcentual, da participação dos parlamentares idosos (50 anos ou mais) em relação ao total de representantes nas esferas municipal, estadual e federal, no Estado de São Paulo.

\begin{tabular}{lccc}
\hline ESTADO SÃO PAULO & Total de representantes & 50 anos + & $\mathbf{\%}$ \\
\hline MUNICIPAL & 74 & 52 & 70,27 \\
\hline ESTADUAL & 96 & 53 & 55,2 \\
\hline FEDERAL & 75 & 46 & 61,34 \\
\hline TOTAL & 245 & 151 & 61,63 \\
\hline Fonte: Elaborada pelos autores $(2020)$ & & &
\end{tabular}

Tabela 8- Distribuição, numérica e porcentual, da participação dos parlamentares idosos (50 anos ou mais) em relação ao total de representantes nas esferas municipal, estadual e federal, na Região Sudeste.

\begin{tabular}{lccc}
\hline SUDESTE & Total de representantes & 50 anos+ & \% \\
\hline MUNICIPAL & 187 & 108 & 57,75 \\
\hline ESTADUAL & 279 & 141 & 50,54 \\
\hline FEDERAL & 193 & 103 & 53,37 \\
\hline TOTAL & 659 & 352 & 53,41 \\
\hline
\end{tabular}

Fonte: Elaborada pelos autores (2020)

Tabela 9- Distribuição, numérica e porcentual, da participação dos parlamentares idosos (50 anos ou mais) em relação ao total de representantes no senado, câmara estadual e federal, na Região Sudeste.

\begin{tabular}{lccc}
\hline SUDESTE & Total de representantes & $\mathbf{5 0}$ anos + & $\mathbf{\%}$ \\
\hline SENADO & 12 & 9 & 75 \\
\hline CÂMARA ESTADUAL & 271 & 136 & 50,18 \\
\hline CÂMARA FEDERAL & 180 & 93 & 51,67 \\
\hline TOTAL & 462 & 237 & 51,30 \\
\hline
\end{tabular}

Fonte: Elaborada pelos autores (2020)

DISCUSSÃO

O Brasil tem presenciado uma mudança demográfica, mediante o declínio nas taxas de fecundidade e aumento da longevidade, em muito pouco tempo. $\mathrm{O}$ envelhecimento da população consiste no aumento do número de idosos e redução na quantidade de jovens, e exige uma análise em longo prazo, do ponto de vista social, político e econômico $^{12}$. Há uma estimativa de que no ano de 2060 exista aproximadamente 58,2 milhões de idosos, um percentual de $25,5 \%$ - um quarto - da população total brasileira ${ }^{3}$.

O envelhecimento ativo e independente, voltado para a integração e a autogestão, constitui um segmento geracional dentro do universo de pessoas consideradas idosas, ou seja, são os "velhos jovens" com idade entre sessenta e oitenta anos. Este indivíduo tem assumido, cada vez mais, papéis sociais importantes, decorrente do aumento da expectativa de vida e da estrutura demográfica brasileira atual ${ }^{1}$. Neste estudo, ao avaliar a participação dos idosos na representação política atual constatou-se uma parcela significativa deste grupo, sendo 30,2\% no Estado de São Paulo e $24,16 \%$ na Região Sudeste. Entre as esferas de atuação, a maior participação dos idosos ocorreu na 
esfera municipal, em ambas análises $(39,19 \%$ SP e $28,88 \%$ Sudeste), embora também tenham participação acima de $20 \%$ nas esferas estaduais e federais. Cumpre destacar a idade mínima de 21 anos para elegibilidade dos deputados e 35 para senadores na legislação brasileira.

Neste estudo, a faixa etária que mostrou a maior concentração de parlamentares foi a de 50 a 59 anos tanto no Estado de São Paulo (31,43\%) quanto na região sudeste $(29,18 \%)$, apesar de não ter sido a com maior número de candidatos na eleição no país. Dados do Tribunal Superior Eleitoral (TSE) mostram que, entre as candidaturas cadastradas para as Eleições 2018, a faixa etária com mais representantes foi a que vai dos 45 aos 49 anos (16,1\% dos candidatos) e depois os candidatos entre 50 e 54 anos, com $15,88 \%$ do total 13 .

Em 2019, nas duas Casas Legislativas, a Câmara e o Senado, $62 \%$ dos parlamentares estão acima dos 50 anos ${ }^{5}$. Resultado semelhante ao observado no estado de São Paulo, com $61,63 \%$ dos parlamentares acima de 50 anos. A maioria dos parlamentares eleita no estado de São Paulo, em 2016 e 2018, está acima de 50 anos, com maior porcentagem na esfera municipal $(70,27 \%)$ seguida pela federal $(61,34 \%)$ e estadual $(55,2 \%)$. O mesmo verificou-se na Região Sudeste $(53,34 \%)$, porém com porcentagens inferiores, nas 3 esferas: municipal $(57,75 \%)$, federal $(52,12 \%)$ e estadual $(50,54 \%)$. Na Câmara, os parlamentares acima de 50 anos representam 59\% em $2019^{5}$, e os da Região Sudeste $51,39 \%$. No Senado, chegam a $80,25 \%{ }^{11}$, e os da Região Sudeste representam $75 \%{ }^{11}$.

Em nossos resultados, considerando a faixa etária de 60 anos ou mais, foi possível verificar que, enquanto no Estado de São Paulo esta é a segunda mais frequente $(30,2 \%)$, na região Sudeste ocupa a terceira posição $(24,16 \%)$, ficando a segunda maior frequência com a faixa etária de 40 a 49 anos $(25,99 \%)$. Nas eleições de 2018, havia cinco candidatos de 90 a 94 anos e dez entre 85 e 89 anos $^{13}$. Entre os deputados estaduais, verificou-se que os idosos representam 25,53\% no estado de São Paulo e 17,71\% na região Sudeste. Entre os deputados estaduais, o mais velho é da Bahia, e se reelegeu deputado estadual aos 81 . O deputado estadual mais jovem do Brasil também foi o mais votado em seu estado, o Ceará, e tem 21 anos, a idade mínima para assumir o cargo ${ }^{14}$.

Lemos e Ranincheski $^{16}$ verificaram estabilidade na composição da Câmara dos Deputados Federais, nos períodos pré-64, militar e pós-88, estando $68 \%$ na faixa dos 40 a 59 anos. Houve redução desta faixa etária para $53,88 \%$ dos eleitos para Câmara dos deputados em 2019, os quais possuem média de idade de 49 anos, e a faixa etária com maior representação é de 51 a 60 anos $(28,26 \%)^{7}$. Neste estudo, verificamos semelhante percentual de deputados federais na faixa etária de 40 a 59 anos entre os representantes do Estado de São Paulo $(55,71 \%)$ e da região Sudeste $(56,98 \%)$. Em 2015, segundo Martins, Calil ${ }^{5}$ (2015) 59\% da Câmara dos Deputados estava acima de 50 anos, sendo semelhante aos nossos resultados dos deputados federais em 2019, com 58,57\% de São Paulo e $51,68 \%$ da Região Sudeste acima de 50 anos. Na Câmara em 2015, o grupo majoritário $(36,84 \%)$ estava na faixa etária entre 51 e 60 anos $^{5}$, e se manteve em 2019 com a maior representação $(28,26 \%)^{7}$. Este estudo também verificou, em 2019, a faixa de 51 a 60 anos como majoritária nos representantes do Estado de São Paulo na Câmara $(34,28 \%)$. Por outro lado, entre os deputados federais da Região Sudeste, em 2019, a faixa etária de 50 a 59 anos $(29,05 \%)$ foi apenas levemente superior à faixa etária de 40 a 49 anos $(27,93 \%)$. Se considerarmos a representação dos deputados federais idosos (60 anos ou mais) em 2019, verificamos $24,28 \%$ em SP e $22,35 \%$ no Sudeste. Na Câmara dos Deputados, os idosos representam 18,52\% dos parlamentares ${ }^{7}$. A análise do perfil médio dos eleitos para compor a Câmara dos Deputados, a partir de 2019, mostra que tem maioria de homens de etnia branca, casados e com ensino superior, idade média de 49 anos variando de 22 a 84 anos $^{16}$. Entre os deputados eleitos em 2018, está uma deputada federal reeleita para o sexto mandato por São Paulo, aos 84 anos, sendo a deputada mais velha eleita e, que terá como colega uma deputada eleita do Paraná, aos 22 anos, a mais jovem integrante da Câmara dos Deputados Federais. ${ }^{14,16}$. Em 2015, o mais novo tinha 23 anos e o mais velho 85 anos, com 10 mandatos de deputado federal ${ }^{5}$. Os representantes mais jovem e mais velho da Câmara são mulheres, diferente de 2015 em que ambos eram homens. A bancada feminina na Câmara dos Deputados em 2019, é composta por 77 mulheres, $15 \%$ das cadeiras, e em 2015, a bancada feminina representava $10 \%$ do Parlamento, com 51 deputadas ${ }^{17}$. Mas ainda fica bem distante do índice de 51,5\% que faz das mulheres a maioria da população brasileira, segundo o Instituto Brasileiro de Geografia e Estatística $(\mathrm{IBGE})^{18}$. Quanto à faixa etária, enquanto a maioria da população tem cerca de 34 anos, a maior parte dos deputados da Câmara (145 - 28,26\%) têm entre 51 e 60 anos $^{7}$.

No Senado, segundo Lemos,Ranincheski $(2002)^{15}$ nos períodos pré-64, militar e pós-88, predominavam os representantes na faixa de 50 a 59 anos que, somados aos parlamentares com mais de 60 anos, eram cerca de $75 \%$ do total de senadores. No Senado em 2019, a média de idade é de 56 anos e grupo majoritário manteve-se de 50 a 59 anos $(33,33 \%)^{8}$. Foi observado neste estudo porcentagem ainda maior desta faixa etária nos representantes da Região Sudeste $(54,54 \%)$, e somados aos com mais de 60 anos são $75 \%$, valor igual ao indicado por Lemos,Ranincheschi (2002) ${ }^{15}$. No Senado em 2015, 
os parlamentares acima dos 50 anos chegam a $87 \%^{5}$, enquanto são $76,54 \%$ em $2019^{8,11}$, indicando o aumento na participação dos mais jovens, de 35 a 49 anos. Fato este corroborado pela informação que os senadores acima de 60 anos representam 37,04\% dos eleitos em $2018^{8,11}$ e o resultado deste estudo verificou idosos em $25 \%$ dos senadores da Região Sudeste. Até julho de 2019, o senador mais velho no Senado tinha 85 anos, do Estado da Paraíba, eleito em 2014 com 81 anos $^{8}$, contudo o primeiro suplente de um deputado de Tocantins tomou posse, e se tornou o senador mais velho da história da Brasil, prestes a completar $91 \operatorname{anos}^{8}$. O curioso é que esta legislatura tem o senador mais novo, de 36 anos, e o mais velho da história do Brasil. Dos dez parlamentares mais idosos do Congresso, Câmara e Senado, em 2019, 6 são representantes eleitos da Região Sudeste ${ }^{17}$.

A idade é muito importante, a participação dos jovens é muito importante, principalmente quando são jovens esclarecidos. Mas, a experiência daqueles que têm mais tempo de parlamento é válida, porque a política é muito complexa, é cheia de riscos e as pessoas mais experientes, logicamente, têm uma vivência maior ${ }^{5}$. Segundo o professor José Alvares Moisés, diretor do Núcleo de Pesquisa de Políticas Públicas da USP (Universidade de São Paulo): "No caso do Senado, isso se justifica. É uma casa destinada a representar os Estados, não a população. Tanto que frequentemente são recrutados exgovernadores, ex-presidentes"

\section{CONCLUSÃO}

Conclui-se que os líderes municipais e estaduais mais envolvidos com a Faculdade de Odontologia de Araçatuba são idosos e que estes são cerca de um terço da representação política no Estado de São Paulo, e um pouco menor na região Sudeste. Os idosos tem um papel atuante na política atual, em especial na esfera municipal analisada do Estado de São Paulo e da região Sudeste, estando entre as faixas etárias com maior representação.

\section{REFERÊNCIAS}

1. Rodrigues LS, Soares GA. Velho, idoso e terceira idade na sociedade contemporânea. Ágora. 2006;4:1-29.

2. Peixoto C. Entre o estigma e a compaixão e os termos classificatórios:velho, velhote, idoso, terceira idade. In: Barros MML de. (Org.). Velhice ou terceira idade? Rio de Janeiro: FGV;1998. p. 69-84

3. Instituto Brasileiro de Geografia e Estatística. Projeção da População 2018: número de habitantes do país deve parar de crescer em 2047. IBGE, Rio de Janeiro, 2018. Disponível em:<https://agenciadenoticias.ibge.gov.br/agencia -sala-de-imprensa/2013-agencia-denoticias/releases/21837-projecao-da-populacao- 2018-numero-de-habitantes-do-pais-deve-pararde-crescer-em-2047>. Acesso em: 08 mai 2020. (tirado de farias 2019)

4. Tribunal Superior Eleitoral. Brasil tem 147,3 milhões de eleitores aptos a votar nas Eleições 2018. TSE, Comunicação, 2018 Ago 01. Disponível

em: $<$ http://www.tse.jus.br/imprensa/noticiastse/2018/Agosto/brasil-tem-147-3-milhoes-deeleitores-aptos-a-votar-nas-eleicoes-2018> Acesso em: 08 jun 2019

5. Martins C, Calil T. Com mais de $60 \%$ de deputados e senadores acima dos 50 anos, Congresso não reflete demandas dos jovens. Notícias R7. 2015 Abr 04. Disponível em: $<$ https://noticias.r7.com/brasil/com-mais-de-60de-deputados-e-senadores-acima-dos-50-anoscongresso-nao-reflete-demandas-dos-jovens04042015> Acesso em 15 de maio de 2020.

6. Tribunal Superior Eleitoral. Estatísticas. TSE, Comunicação, 2018. Disponível em: $<$ http://www.tse.jus.br/eleicoes/estatisticas/est atisticas> Acesso em: 08 jun 2019.

7. Agência Câmara de Notícias. Nova composição da Câmara ainda tem descompasso em relação ao perfil da população brasileira. 2019 Jan 22. Disponível em: <https://www.camara.leg.br/noticias/550900nova-composicao-da-camara-ainda-temdescompasso-em-relacao-ao-perfil-da-populacaobrasileira/>Acesso em 20 de maio de 2020.

8. Agência Senado. Nova bancada tem o senador mais jovem já eleito no Brasil.Da Redação. Agência Senado. 2018 Out 09. ATUALIZADO 2018 Out 09. Disponível em: <https://www12.senado.leg.br/noticias/materias/2 018/10/09/senado-tem-o-senador-mais-jovem-jaeleito-no-brasil> Acesso em 20 de maio de 2020.

9. Gazeta do Povo. Especiais Eleições 2016. Resultados das eleições 2016. Prefeitos, vereadores. Disponível em: $<$ https://especiais.gazetadopovo.com.br/eleico es/2016/> Acesso em fev 2020.

10. Gazeta do Povo. Especiais Eleições 2018. Resultados das eleições 2018:Presidente, Governados, Senador, Deputados Federais, Deputados Estaduais. Disponível em:< https://especiais.gazetadopovo.com.br/eleicoes/20 18/resultados> Acesso em fev 2020.

11. Senado Federal. Senadores em Exercício $56^{\mathrm{a}}$ Legislatura(2019-2023). Disponível em:< https://www25.senado.leg.br/web/senadores/emexercicio/> Acesso em maio 2020.

12. Farias MFRN, Souza PHV de, Santos VÉ da S. O novo retrato demográfico do Brasil: análise acerca do envelhecimento populacional e suas decorrências econômicas. Rev.Bras.de Direito e Gestão Pública. 2019;7:01-11.

13. Dearo G.Os candidatos mais velhos das eleições 
2018:dois tem mais de 100 anos. Exame.2018 Set 01. Disponível em:https://exame.com/brasil/oscandidatos-mais-velhos-das-eleicoes-2018-doistem-mais-de-100-anos/ . Acesso em: 22 mai. 2020

14. Paixão A, Gelape L. Veja quem são os deputados mais novos e mais velhos eleitos em 2018. Portal G1-Globo.2018 Out 08. Disponível em: <https://g1.globo.com/politica/eleicoes/2018/eleic ao-em-numeros/noticia/2018/10/08/veja-quemsao-os-deputados-mais-novos-e-mais-velhoseleitos-em-2018.ghtml> Acesso em 13 de junho de 2020.

15. Lemos LB de S, Ranincheski S. O perfil sociopolítico dos senadores brasileiros. Senatus. 2002;2(1):33-9.

16. Caesar G. Perfil médio do deputado federal eleito é homem, branco, casado e com ensino superior. Portal G1-Globo. 2018 Out 21. Disponível em: <https://g1.globo.com/politica/eleicoes/2018/eleic ao-em-numeros/noticia/2018/10/21/perfil-mediodo-deputado-federal-eleito-e-homem-brancocasado-e-com-ensino-superior.ghtml> Acesso em 15 de maio de 2020.

17. Departamento Intersindical de Assessoria Parlamental-DIAP. Novo congresso nacional em números 2019-2023. Site Diap.org. 2018 Out. Disponível em:<https://static.poder360.com.br/2018/10/Novo -Congresso-Nacional-em-Numeros-20192023.pdf> Acesso em 12 de abril de 2020.

18. Instituto Brasileiro de Geografia e Estatística. Síntese de indicadores socias. Uma análise das condições de vida da população brasileira: 2016. IBGE, Coordenação de População e Indicadores Sociais. Rio de Janeiro: IBGE, 2016.146p. Disponível em: $<$ https://biblioteca.ibge.gov.br/visualizacao/liv ros/liv98965.pdf>

\section{CONFLITO DE INTERESSES}

Os autores declaram não haver conflitos de interesse.

\section{AUTOR PARA CORRESPONDÊNCIA}

\section{Leda Maria Pescinini Salzedas}

leda.salzedas@unesp.br

Submetido em 02/07/2020

Aceito em 10/07/2020 